\title{
Suppression of Natural Convection Heat Transfer Coefficients in an Attic Shaped Enclosure
}

\author{
T.N. Anderson ${ }^{1,2 *}$, M. Duke² and J.K. Carson ${ }^{2}$ \\ ${ }^{1}$ School of Engineering, Deakin University, Geelong VIC 3217, Australia \\ ${ }^{2}$ Department of Engineering, University of Waikato, Hamilton 3240, New Zealand
}

\begin{abstract}
The use of convection suppression devices has been widely discussed in the literature as a means of reducing natural convection heat loss from enclosed spaces. In this study the use of a single baffle was examined as a possible low cost means of suppressing heat loss by natural convection in an attic shaped enclosure.
\end{abstract}

Recently the authors experimentally demonstrated that the natural convection heat transfer for an attic shaped enclosure with Grashof Numbers in the range of $10^{7}$ to $10^{9}$ [1] could be predicted using a correlation in the literature developed for Grashof Numbers in the range $10^{5}$ and $10^{6}$ [2]. However, in this study the impact of adding a single baffle to the enclosure was examined. It was found that the heat transfer coefficient changes with increasing baffle length, and can be predicted using a modified form of the correlation presented in [1], as shown in Eqn. 1.

$$
N u=0.286 A^{-0.286} G r^{0.25}\left(1-\frac{L}{H}\right)^{0.2}
$$

Keywords: triangular enclosure, heat transfer, attic, natural convection, convection suppression

\section{Introduction}

The use of baffles and fins presents a simple and efficient means of altering the heat transfer within an enclosure. The use of baffles in attic shaped enclosures has however been the subject of very few studies. Based on the observations made in the previous studies, it is apparent that the apex between the heated and cooled sides of a triangular or attic shaped enclosure is a point of significant heat transfer.

\footnotetext{
* Corresponding Author: Tel.: +61 35227 3403; Fax: +61 35227 2028;

E-mail address: timothy.anderson@deakin.edu.au (T.N. Anderson)
} 
In their computational studies Moukalled et al [3-6] attempted to modify the flow and heat transfer in a trapezoidal attic style enclosure by including baffles mounted from the floor and inclined sides. They showed that the use of these baffles resulted in a decrease in heat transfer. Similarly, in their computational study Ridouane and Campo [7] showed that attaching baffles to the inclined walls of an isosceles triangular shaped enclosure could also reduce heat transfer.

For porous media filled right triangular enclosures, Varol et al [8] found that an adiabatic fin could be used to control the heat transfer. Most recently however, Anderson et al [9] computationally demonstrated that a single vertical baffle could also be used to suppress heat transfer in a triangular attic shaped enclosure. They found that the heat transfer decreased as the baffle length increased.

Despite the number of computational studies that have shown the possibility of using baffles to influence the heat transfer in attic shaped enclosures, there is a lack of experimental data to support the findings; however this study aims to address this shortcoming.

\section{Experimental Method and Analysis}

To determine the heat transfer by natural convection in a baffled enclosure the experimental apparatus developed by Anderson et al [1] was modified to allow the inclusion of a baffle. As such the enclosure $(707.1 \mathrm{~mm} \times 353.6 \mathrm{~mm} \times 1500 \mathrm{~mm})$ was modified, by the addition of a $20 \mathrm{~mm}$ thick polystyrene baffle; firstly extending from the apex of the enclosure downwards and secondly from the centre of the base upwards (Figures 1 and 2).

As noted in [1], a flexible aluminium foil resistance heater (750W nominal) was attached to a $2 \mathrm{~mm}$ aluminium plate (one of the inclined sides), and was used to create a temperature gradient within the enclosure. Aluminium plate was used to ensure temperature uniformity on the heated surface inside. Heat loss was minimised from the heater by insulating it with $100 \mathrm{~mm}$ of mineral wool fibre insulation (R2.2) backed with a sheet of $20 \mathrm{~mm}$ plywood.

The second inclined surface was also made from $2 \mathrm{~mm}$ aluminium plate and was cooled by a fan providing a free stream velocity of approximately $4 \mathrm{~m} / \mathrm{s}$ to ensure minimal thermal resistance. This step was done to ensure that the majority of the heat transfer away from the enclosure occurred from this surface. 
For full details of the apparatus and the analysis however, the reader is referred to the previous work [1].

\section{Results}

Based on the experimental methodology and analysis of the natural convection heat transfer in the enclosure, it was possible to determine the heat transfer coefficients over a range of conditions.

In this study the power was varied such that the surface temperature of the heated side was between $30^{\circ} \mathrm{C}$ and $120^{\circ} \mathrm{C}$ under steady state conditions. By recording the steady state data it was then possible to determine the heat transfer coefficient. Taking the vertical height of the enclosure as the characteristic length [9], and the physical properties at the mean enclosure temperature, this resulted in Grashof Numbers in the range of $10^{7}$ to $10^{9}$.

For this study baffles extending $50 \%$ and $75 \%$ of the enclosure height were examined using two configurations: apex and base mounted. Further, it should be noted that the addition of a baffle did not change the way in which the Grashof Number was defined or calculated.

In Figure 3 it can be seen that as in the non-baffled enclosure condition, there is an increase in the heat transfer coefficient in the baffled enclosure with an increased temperature gradient. However it can also be seen that the inclusion of the apex mounted baffle has resulted in a suppression of the natural convection heat transfer coefficients. Similarly in Figure 4 it can be seen that by transferring the baffle from the apex to the base, such that it extends upwards rather than downwards, there is also a reduction in the heat transfer coefficient.

On closer inspection of Figures 3 and 4 it can be seen that there is negligible difference between the heat transfer coefficients of the apex or base mounted baffles. From this it is apparent that the location of the baffle, when it is mounted under the apex of the enclosure, has essentially no impact on heat transfer and that rather it is dictated purely by the length of the baffle.

Now if we consider the influence that the baffle has on the existing correlation reported in [1], it can be seen in Figure 5 that using this correlation, and thereby disregarding the baffle, would result in an over-prediction of the heat transfer coefficients. Therefore, in order to account for the presence of the baffle, it was decided to modify the existing correlation. 
To do this a non-dimensional length parameter (referred to as the blockage ratio) represented by the ratio of the baffle length $(\mathrm{L})$ to the enclosure height $(\mathrm{H})$ was added to the correlation, as shown in Eqn. 2. It was found that this correlation provided good correlation with the experimental data, both with and without a baffle as shown in Figure 6.

$$
N u=0.286 A^{-0.286} G r^{0.25}\left(1-\frac{L}{H}\right)^{0.2}
$$

It should however be noted that Eqn. 2, and the method used to derive it, cannot be applied when the blockage ratio is equal to unity. Using the methodology described previously, the Nusselt number will equal zero thus implying an adiabatic chamber, and in reality this cannot occur. Rather by increasing the length of the baffle so the blockage ratio is equal to one, the enclosure becomes two right triangular enclosures separated by a partition. Therefore alternative correlations would need to be used to determine the heat loss and the thermal resistance of the partition would also need to be considered.

\section{Conclusion}

In the authors' previous study [1] it was noted that a correlation developed by Ridouane and Campo'[2] to describe natural convection in attic style enclosures for Grashof Numbers in the range $2.9 \times 10^{6}$ to $9 \times 10^{6}$ was also valid for Grashof Numbers in the range of $10^{7}$ to $10^{9}$. This served to validate the proposition of Anderson et al $[9,10]$ that natural convection in the attic of a cold roof building could serve as an insulating medium for roof integrated solar collectors installed in a cold roof building.

In this study it has been shown that this effect could potentially be enhanced by the addition of a single baffle, mounted either from the apex down or the base up, in an attic shaped enclosure. Here it has been demonstrated that the addition of a baffle serves to suppress the heat transfer and can be predicted by an original correlation that relates not only to the proportions of the enclosure but also to the non-dimensional blockage ratio.

\section{References}

[1] T.N. Anderson, M. Duke, J.K. Carson, Experimental determination of natural convection heat transfer coefficients in an attic shaped enclosure, International Communications in Heat and Mass Transfer 37 (4) (2010) 360-363 
[2] E.H. Ridouane, A. Campo, Experimental-based correlations for the characterization of free convection of air inside isosceles triangular cavities with variable apex angles, Experimental Heat Transfer 18 (2) (2005) 81-86

[3] F. Moukalled, S. Acharya, Natural Convection in a Trapezoidal Enclosure with Offset Baffles, Journal of Thermophysics and Heat Transfer 15 (2) (2001) 212-218

[4] F. Moukalled, S. Acharya, Buoyancy-induced heat transfer in partially divided trapezoidal cavities, Numerical Heat Transfer, Part A: Applications 32 (8) (1997) 787-810

[5] F. Moukalled, S. Acharya, Natural convection in trapezoidal cavities with baffles mounted on the upper inclined surfaces, Numerical Heat Transfer, Part A: Applications 37, (6) (2000) 545-565

[6] F. Moukalled, M. Darwish, Natural Convection in a Trapezoidal Enclosure Heated from the Side with a Baffle Mounted on Its Upper Inclined Surface, Heat Transfer Engineering 25 (8) (2004) 80-93

[7] E.H. Ridouane, A. Campo, Effects of attaching baffles onto the inclined walls of attic frames for purposes of energy conservation, Heat Transfer Engineering 28 (2) (2007) 103-111 [8] Y. Varol, H.F. Oztop, A. Varol, Natural convection in porous triangular enclosures with a solid adiabatic fin attached to the horizontal wall, International Communications in Heat and Mass Transfer 34 (1) (2007) 19-27

[9] T.N. Anderson, M. Duke, J.K. Carson, Convection suppression in a triangular-shaped enclosure, Computational Thermal Sciences 1 (3) (2009) 309-321

[10] T.N. Anderson, M. Duke, G.L. Morrison, J.K. Carson, Performance of a Building Integrated Photovoltaic/Thermal (BIPVT) Solar Collector, Solar Energy 83 (4) (2009) 445-455 


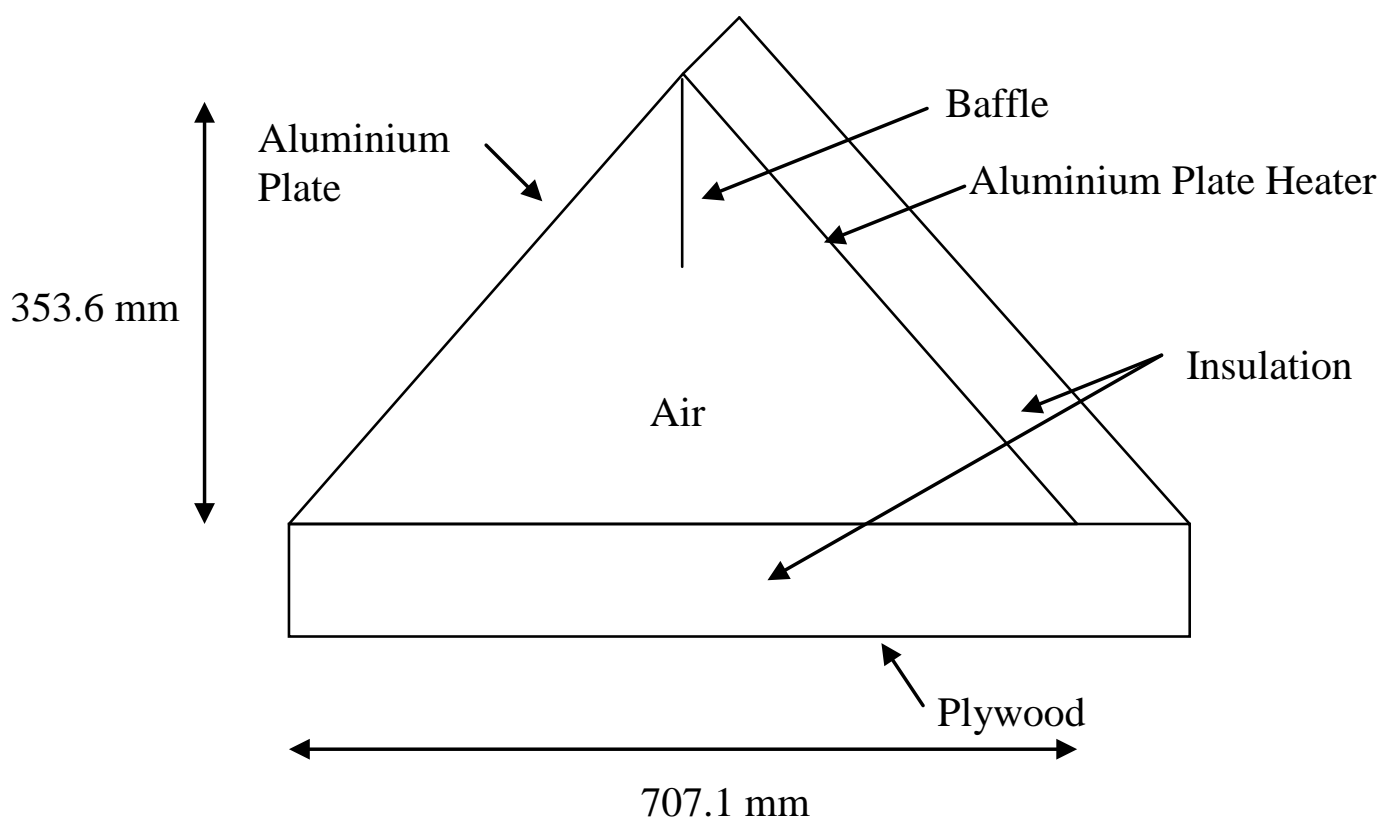

Fig. 1: Layout of experimental enclosure with top mounted baffle 


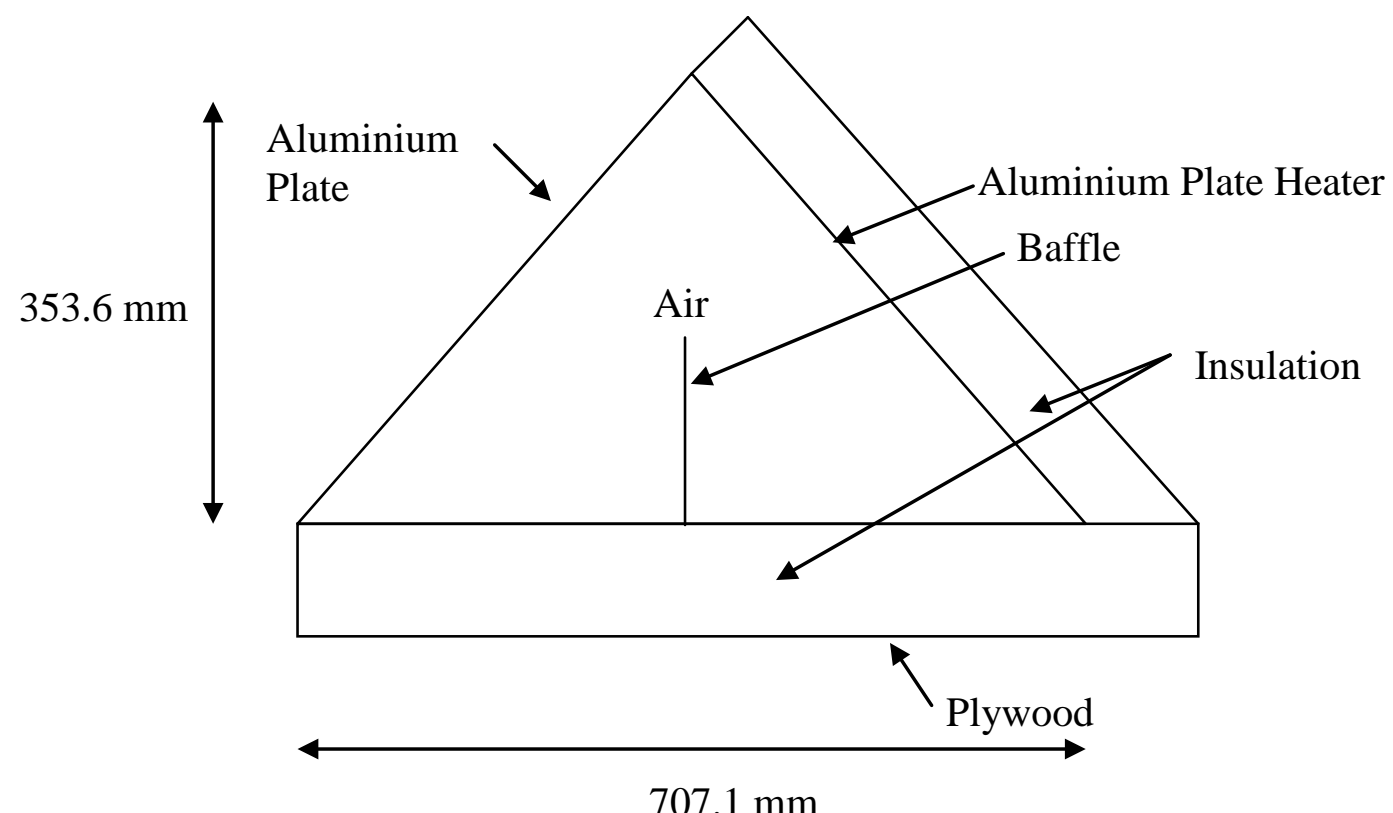

Fig. 2: Layout of experimental enclosure with base mounted baffle 


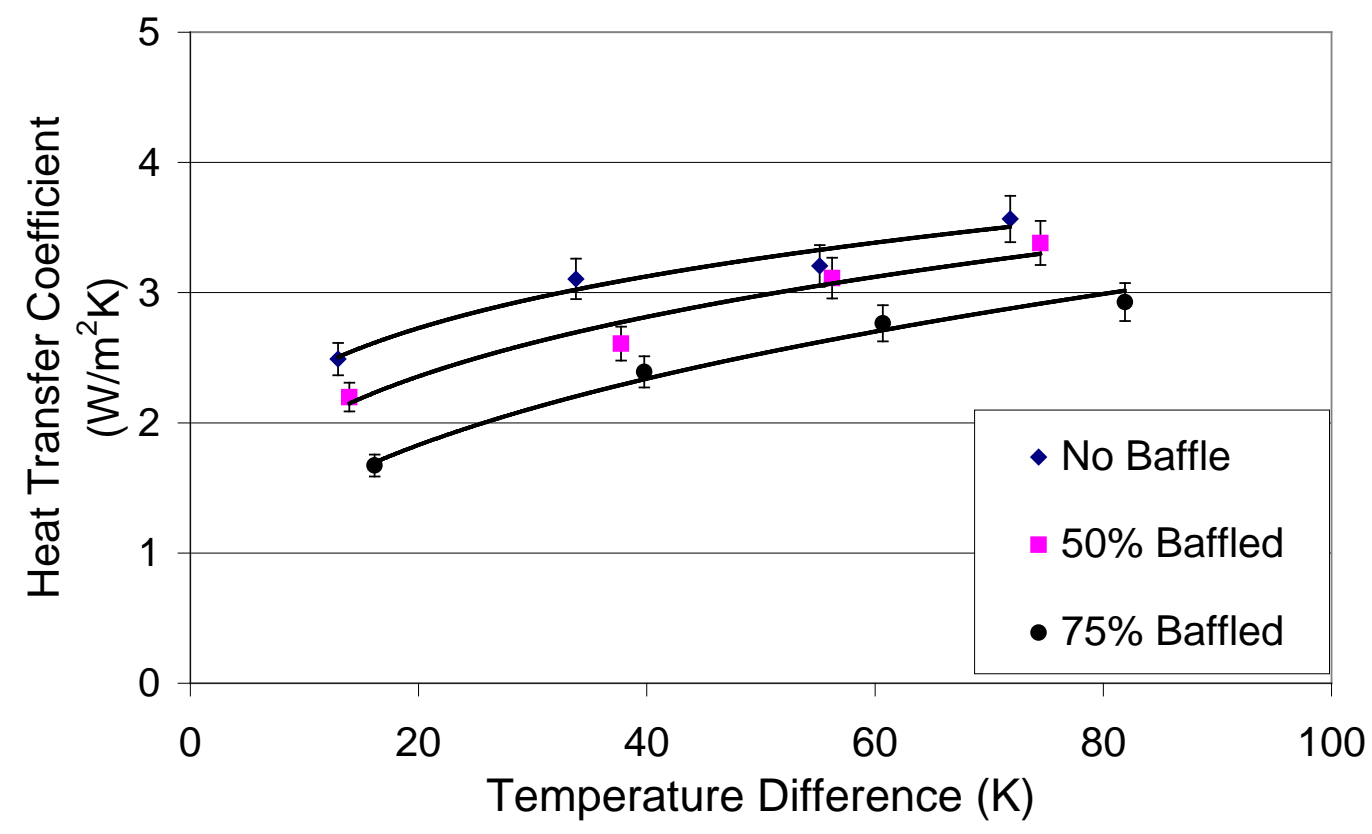

Fig. 3: Change in experimental heat transfer coefficient for apex mounted baffle 


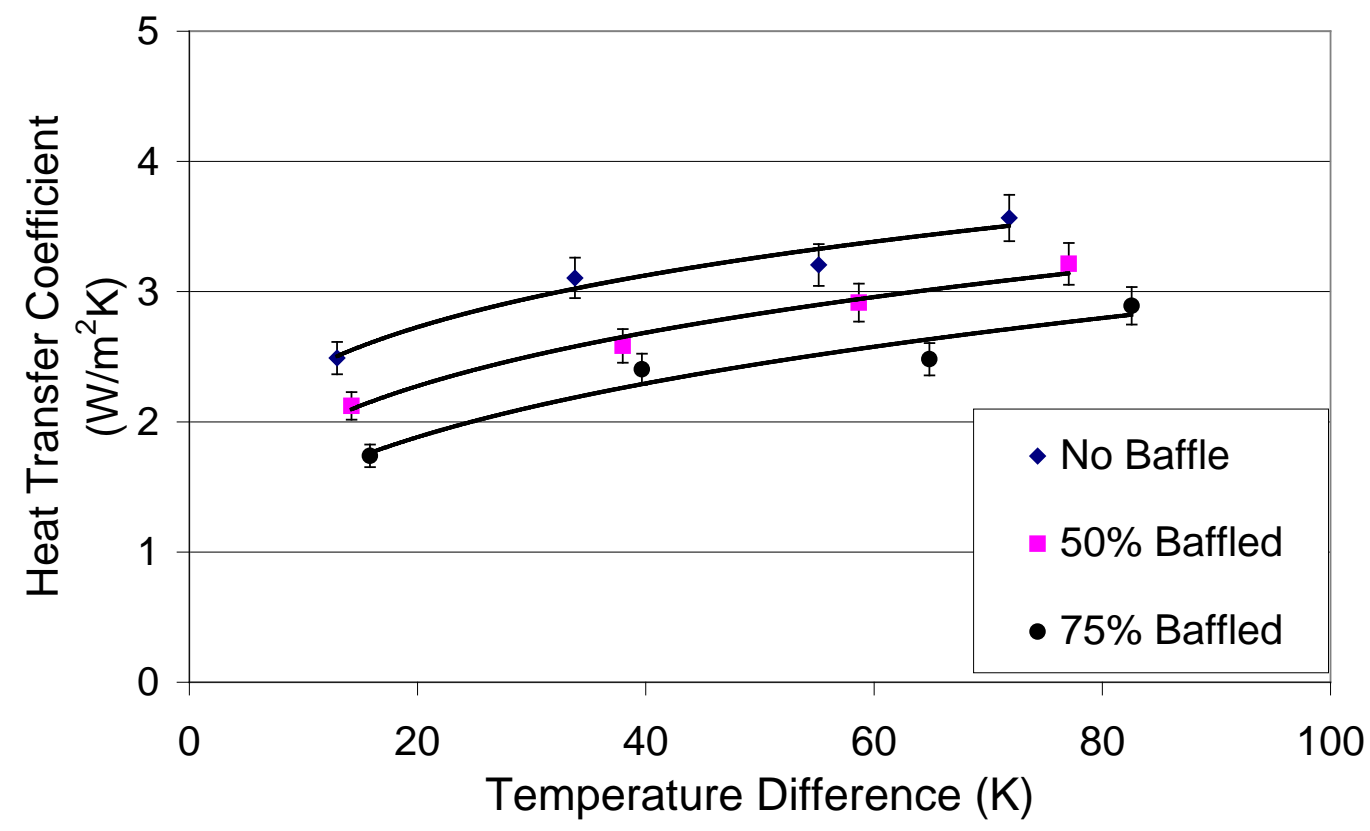

Fig. 4: Change in experimental heat transfer coefficient for base mounted baffle 


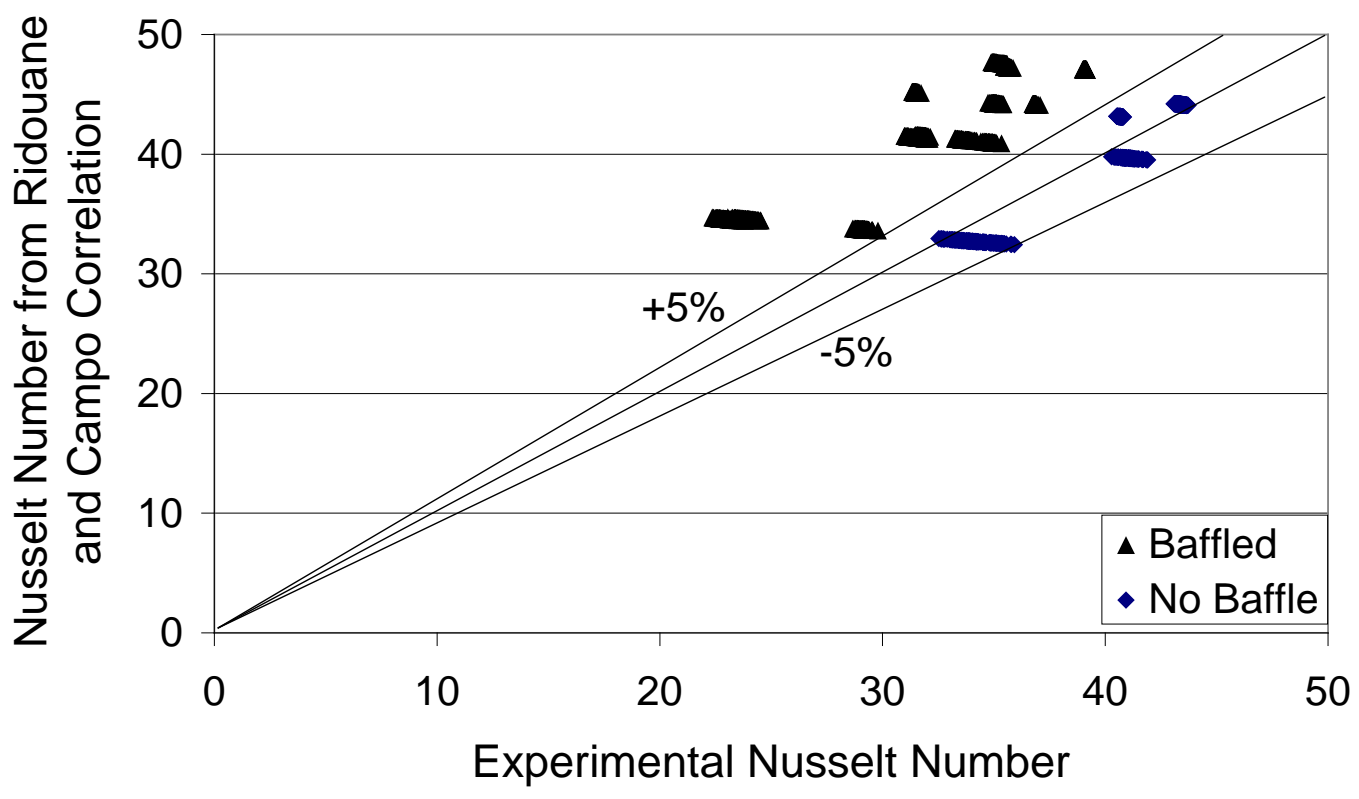

Fig. 5: Nusselt number in a baffled enclosure, showing over-prediction by Ridouane and Campo's correlation 


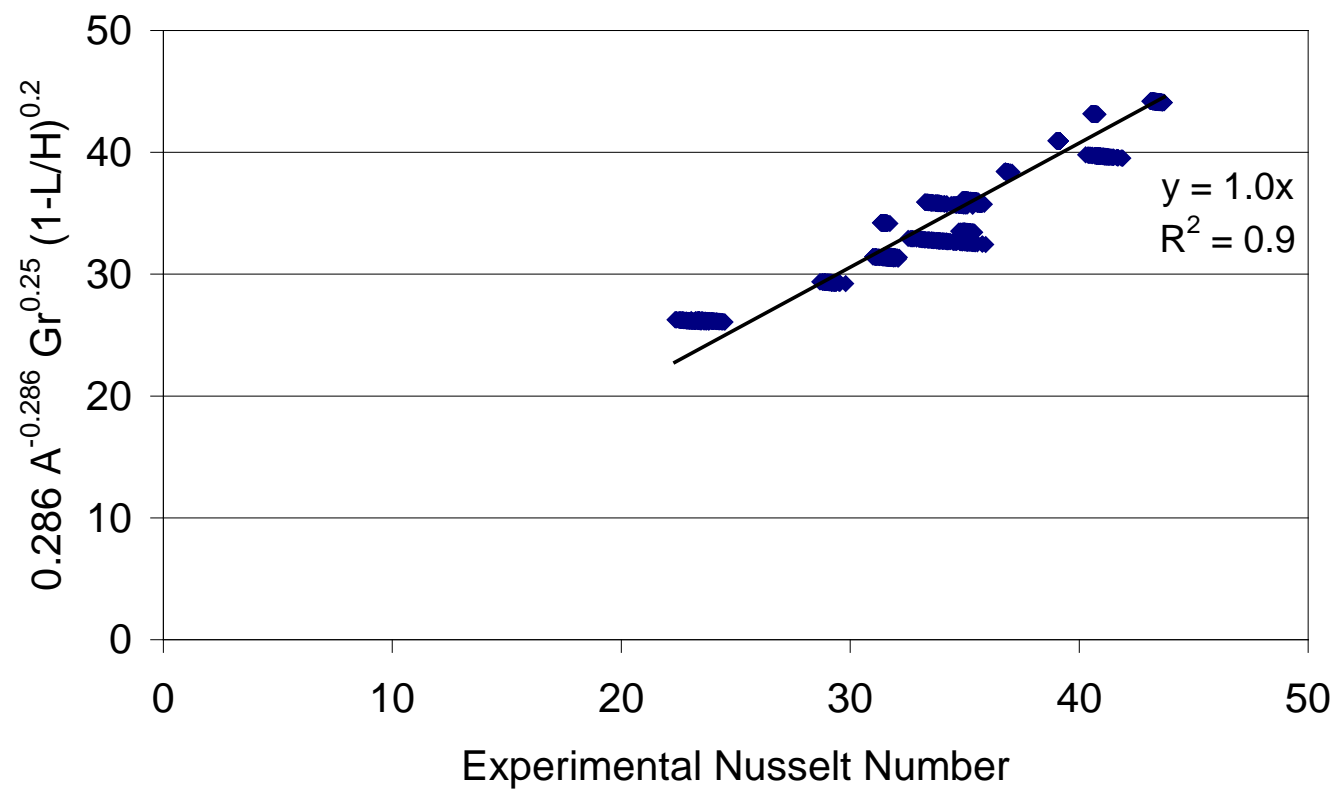

Fig. 6: Experimental Nusselt number versus baffled enclosure correlation 